\title{
Invariance of Stationary and Ergodic Properties of a Quantum Source under Memoryless Transformations*
}

\author{
Alexei Kaltchenko ${ }^{1 \dagger}$ and En-Hui Yang ${ }^{2}$ \\ 1,2 E\&CE Department, University of Waterloo, \\ Waterloo, Ontario N2L 3G1, Canada
}

February 26, 2019

\begin{abstract}
We prove that the stationarity and the ergodicity of a quantum source $\left\{\rho_{[1, m]}\right\}_{m=1}^{\infty}$ are preserved by any trace-preserving completely positive linear map of the tensor product form $\mathcal{E}^{\otimes m}$, where a copy of $\mathcal{E}$ acts locally on each spin lattice site. We also establish ergodicity criteria for so called classically-correlated quantum sources.
\end{abstract}

\section{Introduction}

The quantum ergodicity is as instrumental in studying quantum information systems as is the classical ergodicity in studying classical information systems. To give a rough idea of the role that quantum ergodicity plays in quantum information theory, one may name just one result, the quantum extension [3] of the Shannon-McMillan theorem which implies that a quantum stationary ergodic source can be compressed up to its von Neumann entropy rate.

In this paper we are concerned with stationary and ergodic properties of quantum sources. Specifically, we study the case when a stationary and ergodic (weakly mixing or strongly mixing, respectively) quantum source $\left\{\rho_{[1, m]}\right\}_{m=1}^{\infty}$ is subjected to a trace-preserving completely positive linear transformation (map) of the tensor product form $\mathcal{E}^{\otimes m}$, where a copy of $\mathcal{E}$ locally acts on each spin lattice site. We present several technical lemmas and prove that the map preserves all the listed source properties. Such maps describe the effect of a transmission via a memoryless channel as well as the effect of memoryless coding, both lossless and lossy ones. As a corollary of our main result, we also establish ergodicity criteria for so called classically-correlated quantum sources.

\footnotetext{
${ }^{*}$ This work was supported in part by the Natural Sciences and Engineering Research Council of Canada under Grants RGPIN203035-98 and RGPIN203035-02, by the Premier's Research Excellence Award, by the Canada Foundation for Innovation, by the Ontario Distinguished Research Award, and by the Canada Research Chairs Program.

${ }^{\dagger}$ e-mail: akaltche@bbcr.uwaterloo.ca
} 


\section{Quantum Sources: Mathematical Formalism and Notation}

Informally, a quantum source is a triple[6] consisting of quantum messages, a probability distribution for the messages, and the time shift. Such the triple uniquely determines a state of a one-dimensional quantum lattice system. While any given state corresponds to infinitely many different quantum sources, the state formalism essentially captures all the information-theoretic properties of a corresponding quantum source. Thus, the notion of "quantum source" is usually identified with the notion of "state" of the corresponding lattice system and used interchangeably.

Suppose $Q$ is an infinite quantum spin lattice system over lattice $\mathbb{Z}$ of integers. To describe $Q$, we use the standard mathematical formalism introduced in [4] and [10] and borrow the notation from [3], [6], and [9]. Let $\mathfrak{A}$ be a $C^{*}$-algebra with identity of the bounded operators $\mathcal{B}(\mathcal{H})$ on a $d$ dimensional Hilbert space $\mathcal{H}, d<\infty$. To each $\mathbf{x} \in \mathbb{Z}$ there is associated an algebra $\mathfrak{A}_{\mathbf{x}}$ of observables for a spin located at site $\mathbf{x}$, where $\mathfrak{A}_{\mathbf{x}}$ is isomorphic to $\mathfrak{A}$ for every $\mathbf{x}$. The local observables in any finite subset $\Lambda \subset \mathbb{Z}$ are those of the finite quantum system

$$
\mathfrak{A}_{\Lambda}:=\bigotimes_{\mathbf{x} \in \Lambda} \mathfrak{A}_{\mathbf{x}}
$$

The quasilocal algebra $\mathfrak{A}_{\infty}$ is the operator norm completion of the normed algebra $\bigcup_{\Lambda \subset \mathbb{Z}} \mathfrak{A}_{\Lambda}$, the union of all local algebras $\mathfrak{A}_{\Lambda}$ associated with finite intervals $\Lambda \subset \mathbb{Z}$. A state of the infinite spin system is given by a normed positive functional

$$
\varphi: \mathfrak{A}_{\infty} \rightarrow \mathbb{C}
$$

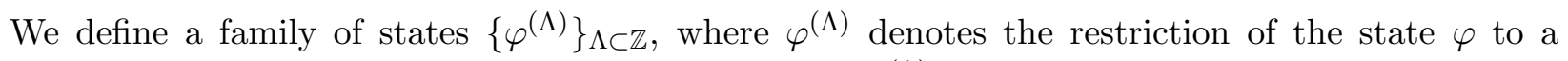
finite-dimensional subalgebra $\mathfrak{A}_{\Lambda}$, and assume that $\left\{\varphi^{(\Lambda)}\right\}_{\Lambda \subset \mathbb{Z}}$ satisfies the so called consistency condition 3 , 6], that is

$$
\varphi^{(\Lambda)}=\varphi^{\left(\Lambda^{\prime}\right)} \uparrow \mathfrak{A}_{\Lambda}
$$

for any $\Lambda \subset \Lambda^{\prime}$. The consistent family $\left\{\varphi^{(\Lambda)}\right\}_{\Lambda \subset \mathbb{Z}}$ can be thought of as a quantum-mechanical counterpart of a consistent family of cylinder measures. Since there is one-to-one correspondence

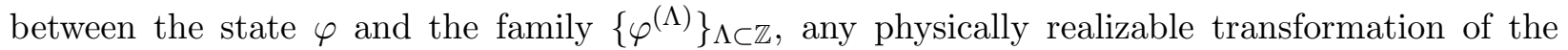
infinite system $Q$, including coding and transmission of quantum messages, can be well formulated using the states $\varphi^{(\Lambda)}$ of finite subsystems.

Let $\gamma$ denote a transformation on $\mathfrak{A}_{\infty}$ which is induced by the right shift on the set $\mathbb{Z}$. Now we are equipped to define the notions of stationarity and ergodicity of a quantum source.

Definition 2.1 A state $\varphi$ is called stationary if $\varphi \circ \gamma=\varphi$.

Definition 2.2 A stationary state $\varphi$ is called ergodic (for a given $\gamma$ ) if it is an extremal point in the set of stationary states.

The following lemma [4, 6, 10] provides a practical method of demonstrating the ergodicity of a state: 
Lemma 2.1 A stationary state $\varphi$ on $\mathfrak{A}_{\infty}$ is ergodic if and only if it satisfies the equality

$$
\lim _{n \rightarrow \infty} \frac{1}{n} \sum_{i=1}^{n} \varphi\left(a \gamma^{i}(b)\right)=\varphi(a) \varphi(b), \quad \forall a, b \in \mathfrak{A}_{\infty},
$$

where $\gamma^{i}$ denotes the right $i$-shift $\underbrace{\gamma \circ \cdots \circ \gamma}_{i}$.

Now we state a series of definitions[5] which provide "stronger" notions of ergodicity:

Definition 2.3 A state $\varphi$ is completely ergodic if it is ergodic for all $\tilde{\gamma} \in\left\{\gamma^{n}: n \geqslant 1\right\}$.

Definition 2.4 A stationary state $\varphi$ on $\mathfrak{A}_{\infty}$ is called weakly mixing if

$$
\lim _{n \rightarrow \infty} \frac{1}{n} \sum_{i=1}^{n}\left|\varphi\left(a \gamma^{i}(b)\right)-\varphi(a) \varphi(b)\right|=0, \quad \forall a, b \in \mathfrak{A}_{\infty} .
$$

Definition 2.5 A stationary state $\varphi$ on $\mathfrak{A}_{\infty}$ is called strongly mixing if

$$
\lim _{i \rightarrow \infty} \varphi\left(a \gamma^{i}(b)\right)=\varphi(a) \varphi(b), \quad \forall a, b \in \mathfrak{A}_{\infty}
$$

\section{Main Result}

In this section we present a sequence of technical lemmas which will lead to our main result. But first we shall reformulate the stationary ergodic properties of an infinite spin lattice system in terms of its finite subsystems. It is known that for each $\varphi^{(\Lambda)}$ there exists a unique density operator $\rho_{\Lambda} \in \mathfrak{A}_{\Lambda}$, such that $\varphi^{(\Lambda)}(a)=\operatorname{tr}_{\Lambda} \rho_{\Lambda} a, a \in \mathfrak{A}_{\Lambda}$ and $\operatorname{tr}_{\Lambda}$ is the trace on $\mathfrak{A}_{\Lambda}$. Thus, any given state $\varphi$ corresponds one-to-one to a consistent family of density operators $\left\{\rho_{[1, m]}\right\}_{m=1}^{\infty}$, and a quantum source will be identified with such a family through the rest of the paper. By rewriting the consistency condition (1), Definition 2.1, and the equations (2-14) in terms of density operators, we obtain the following three elementary lemmas

Lemma 3.1 A family $\left\{\rho_{[1, m]}\right\}_{m=1}^{\infty}$ on $\mathfrak{A}_{\infty}$ is consistent if and only if, for all positive integers $m, i<$ $\infty$ and every $a \in \mathfrak{A}_{[1, m]}$, the following holds:

$$
\operatorname{tr}\left(\rho_{[1, m]} a\right)=\operatorname{tr}\left(\rho_{[1, m+i]}\left(a \otimes I^{\otimes i}\right)\right)
$$

where $I^{\otimes i}$ stands for the $i$-fold tensor product of the identity operators acting on respective spins.

\footnotetext{
${ }^{1}$ In what follows we abusively use the same symbol to denote both an operator (or superoperator), confined to a lattice interval $[1, m]$, and its "shifted" copy, confined to $[1+j, m+j]$, where the value of integer $j$ will be understood from the context.
} 
Lemma 3.2 A quantum source $\left\{\rho_{[1, m]}\right\}_{m=1}^{\infty}$ on $\mathfrak{A}_{\infty}$ is stationary if and only if, for all positive integers $m, i<\infty$ and every $a \in \mathfrak{A}_{[1, m]}$, the following equality is satisfied:

$$
\operatorname{tr}\left(\rho_{[1, m]} a\right)=\operatorname{tr}\left(\rho_{[1, m+i]}\left(I^{\otimes i} \otimes a\right)\right)
$$

Lemma 3.3 A stationary quantum source $\left\{\rho_{[1, m]}\right\}_{m=1}^{\infty}$ on $\mathfrak{A}_{\infty}$ is ergodic (weakly mixing or strongly mixing, respectively) if and only if, for every positive integer $m<\infty$ and all $a, b \in \mathfrak{A}_{[1, m]}$, the equality $\left(2^{*}\right)\left(\left[3^{*}\right)\right.$ or $\left(\mathbb{1}^{*}\right)$, respectively $)$ holds:

$$
\begin{aligned}
& \lim _{n \rightarrow \infty} \frac{1}{n} \sum_{i=m}^{n} \operatorname{tr}\left(\rho_{[1, m+i]}\left(a \otimes I^{\otimes(i-m)} \otimes b\right)\right)=\operatorname{tr}\left(\rho_{[1, m]} a\right) \operatorname{tr}\left(\rho_{[1, m]} b\right), \\
& \lim _{n \rightarrow \infty} \frac{1}{n} \sum_{i=m}^{n}\left|\operatorname{tr}\left(\rho_{[1, m+i]}\left(a \otimes I^{\otimes(i-m)} \otimes b\right)\right)-\operatorname{tr}\left(\rho_{[1, m]} a\right) \operatorname{tr}\left(\rho_{[1, m]} b\right)\right|=0, \\
& \lim _{i \rightarrow \infty} \operatorname{tr}\left(\rho_{[1, m+i]}\left(a \otimes I^{\otimes(i-m)} \otimes b\right)\right)=\operatorname{tr}\left(\rho_{[1, m]} a\right) \operatorname{tr}\left(\rho_{[1, m]} b\right),
\end{aligned}
$$

Before we can state our main result, we need to fix some additional notation. Let $\mathcal{E}$ be an arbitrary trace-preserving quantum operation that has the input space $\mathcal{B}(\mathcal{H})$. Without loss of generality we assume that the output space for $\mathcal{E}$ is also $\mathcal{B}(\mathcal{H})$. It is known $[7$ that $\mathcal{E}$ is a tracepreserving completely positive linear (TPCPL) map. Next, we define a composite map

$$
\mathcal{E}^{\otimes m}: \mathfrak{A}_{[1, m]} \rightarrow \mathfrak{A}_{[1, m]}, \quad \forall m>0 .
$$

We point out that such a tensor product map is the most general description of a quantum memoryless channel[迎.

Theorem 3.1 If $\left\{\rho_{[1, m]}\right\}_{m=1}^{\infty}$ is a stationary and ergodic (weakly mixing or strongly mixing, respectively) source, then so is $\left\{\mathcal{E}^{\otimes m}\left(\rho_{[1, m]}\right)\right\}_{m=1}^{\infty}$.

Proof of Theorem 3.1:

For any TPCPL map there exists a so-called "operator-sum representation" [1], [0]. Then, an $m$-fold tensor product map $\mathcal{E}^{\otimes m}$ has the following representation:

$$
\mathcal{E}^{\otimes m}\left(\rho_{[1, m]}\right)=\sum_{j_{1}, j_{2}, \ldots, j_{m}}\left(A_{j_{1}} \otimes A_{j_{2}} \otimes \cdots \otimes A_{j_{m}}\right) \rho_{[1, m]}\left(A_{j_{1}} \otimes A_{j_{2}} \otimes \cdots \otimes A_{j_{m}}\right)^{\dagger}
$$

with

$$
\sum_{i} A_{i}^{\dagger} A_{i}=I, \quad A_{i}, I \in \mathfrak{A}
$$

where $I$ stands for identity operator.

Due to (7) and (8), the following three equalities hold for all positive integers $m<i<\infty$ and all 
$a, b \in \mathfrak{A}_{[1, m]}$

$$
\begin{aligned}
& \operatorname{tr}\left(\mathcal{E}^{\otimes(m+i)}\left(\rho_{[1, m+i]}\right)\left(a \otimes I^{\otimes(i-m)} \otimes b\right)\right)=\operatorname{tr}\left(\rho_{[1, m+i]}\left(\tilde{a} \otimes I^{\otimes(i-m)} \otimes \tilde{b}\right)\right), \\
& \operatorname{tr}\left(\mathcal{E}^{\otimes m}\left(\rho_{[1, m]}\right) a\right)=\operatorname{tr}\left(\rho_{[1, m]} \tilde{a}\right) \\
& \operatorname{tr}\left(\mathcal{E}^{\otimes m}\left(\rho_{[1, m]}\right) b\right)=\operatorname{tr}\left(\rho_{[1, m]} \tilde{b}\right)
\end{aligned}
$$

where $a, b \in \mathfrak{A}_{[1, m]}$ and $\tilde{a}$ and $\tilde{b}$ are defined as follows:

$$
\begin{aligned}
\tilde{a} & :=\sum_{j_{1}, j_{2}, \ldots, j_{m}}\left(A_{j_{1}} \otimes A_{j_{2}} \otimes \cdots \otimes A_{j_{m}}\right)^{\dagger} a\left(A_{j_{1}} \otimes A_{j_{2}} \otimes \cdots \otimes A_{j_{m}}\right), \\
\tilde{b} & :=\sum_{j_{1}, j_{2}, \ldots, j_{m}}\left(A_{j_{1}} \otimes A_{j_{2}} \otimes \cdots \otimes A_{j_{m}}\right)^{\dagger} b\left(A_{j_{1}} \otimes A_{j_{2}} \otimes \cdots \otimes A_{j_{m}}\right) .
\end{aligned}
$$

Combining (9) with Lemma 3.3, we obtain the ergodicity (weakly mixing or strongly mixing, respectively) of $\left\{\mathcal{E}^{\otimes m}\left(\rho_{[1, m]}\right)\right\}_{m=1}^{\infty}$. In a similar manner, the application of Lemma 3.1 establishes the consistency of $\left\{\mathcal{E}^{\otimes m}\left(\rho_{[1, m]}\right)\right\}_{m=1}^{\infty}$, and the application of Lemma 3.2 establishes the stationarity of $\left\{\mathcal{E}^{\otimes m}\left(\rho_{[1, m]}\right)\right\}_{m=1}^{\infty}$.

Remark 1 We note that any weakly or strongly mixing quantum source is also completely ergodic. Then, for such sources, the theorem trivially extends to cover TPCPL maps of the form $\left(\mathcal{E}^{k}\right)^{\otimes(m / k)}$, $(m / k) \in \mathbb{Z}$, where $\mathcal{E}^{k}$ acts on $k$-blocks of lattice, in direct analogy with a $k$-block classical coding. Thus, our work is the quantum generalization of a well-known classical information-theoretic result [2, Chapter 7] for memoryless-and block-coding and channel transmission.

Definition 3.1 We define a classically correlated quantum source $\left\{\rho_{[1, m]}^{c l s}\right\}_{m=1}^{\infty}$ by an equation

$$
\rho_{[1, m]}^{c l s}:=\sum_{x_{1}, x_{2}, \ldots, x_{m}} p\left(x_{1}, x_{2}, \ldots, x_{m}\right)\left|x_{1}\right\rangle\left\langle x_{1}|\otimes| x_{2}\right\rangle\left\langle x_{2}|\otimes \cdots \otimes| x_{m}\right\rangle\left\langle x_{m}\right|,
$$

where $p(\cdot)$ stands for a probability distribution, and for every $i,\left|x_{i}\right\rangle$ belongs to some fixed linearlyindependent set $S:=\left\{\left|\psi_{1}\right\rangle,\left|\psi_{2}\right\rangle, \ldots,\left|\psi_{d}\right\rangle\right\}$ of vectors in the Hilbert space $\mathcal{H}$. We recall that $\mathcal{H}$ is the support space for the operators in $\mathfrak{A}$. The set $S$ is sometimes called a quantum alphabet.

Corollary 3.2 If a classical probability distribution $p(\cdot)$ in Definition 3.1 is a stationary and ergodic (weakly mixing or strongly mixing, respectively), then so is the quantum source $\left\{\rho_{[1, m]}^{\text {cls }}\right\}_{m=1}^{\infty}$.

\section{Proof of Corollary 3.2:}

Let $S_{\perp}:=\left\{\left|e_{1}\right\rangle,\left|e_{2}\right\rangle, \ldots,\left|e_{d}\right\rangle\right\}$ be any orthonormal basis in $\mathcal{H}$, and let $\left\{\tilde{\rho}_{[1, m]}^{c l s}\right\}_{m=1}^{\infty}$ be the source with alphabet $S_{\perp}$ and distribution $p(\cdot)$. For $i=1, \ldots, d$, we define a set $\left\{A_{i}\right\}$ of linear operators as follows

$$
A_{i}:=\left|\psi_{i}\right\rangle\left\langle e_{i}\right|
$$


Then, set $\left\{A_{i}\right\}$ satisfies (8), and we define a TPCPL map $\mathcal{E}^{\otimes m}$ as in (7). Consequently, we have $\left(\rho_{[1, m]}^{c l s}\right)=\mathcal{E}^{\otimes m}\left(\tilde{\rho}_{[1, m]}^{c l s}\right)$. Thus, to complete the proof, we need to show that $\left\{\tilde{\rho}_{[1, m]}^{c l s}\right\}_{m=1}^{\infty}$ on $\mathfrak{A}_{\infty}$ is ergodic (weakly mixing or strongly mixing, respectively).

Let $\mathfrak{C}$ be a subalgebra of $\mathfrak{A}$ spanned by the set $\left\{\left|e_{i}\right\rangle\left\langle e_{i}|:| e_{i}\right\rangle \in S_{\perp}\right\}$. We extend $\mathfrak{C}$ to a quasilocal algebra $\mathfrak{C}_{\infty} \subset \mathfrak{A}_{\infty}$ over lattice $\mathbb{Z}$ in the same way we did for $\mathfrak{A}_{\infty}$. The algebra $\mathfrak{A}_{\infty}$ is clearly abelian due to the orthogonality of the set $S_{\perp}$. By our construction, $\tilde{\rho}_{[1, m]}^{c l s}$ is an element of algebra $\mathfrak{C}_{[1, m]} \subset \mathfrak{A}_{[1, m]}$ for every $m$. Therefore, combining Lemma 3.3 with [8, Theorem 2.2], we conclude that the ergodicity (weakly mixing or strongly mixing, respectively) of the source $\left\{\tilde{\rho}_{[1, m]}^{c l s}\right\}_{m=1}^{\infty}$ on $\mathfrak{A}_{\infty}$ is equivalent to its ergodicity (weakly mixing or strongly mixing, respectively) on $\mathfrak{C}_{\infty}$, Finally, we note that since $\mathfrak{C}_{\infty}$ is abelian, $\left\{\tilde{\rho}_{[1, m]}^{c l s}\right\}_{m=1}^{\infty}$ on $\mathfrak{C}_{\infty}$ is just a classical stochastic process with distribution $p(\cdot)$.

\section{References}

[1] H. Barnum, E. Knill, and M. Nielsen, "On quantum fidelities and channel capacities", IEEE Trans. Inform. Theory, Vol. 46, No. 4, pp. 1317-1329, July, 2000.

[2] T. Berger, Rate distortion theory; a mathematical basis for data compression, Englewood Cliffs, N.J., Prentice-Hall, 1971.

[3] I. Bjelakovic, T. Krueger, R. Siegmund-Schultze, A. Szkola, "The ShannonMcMillan Theorem for Ergodic Quantum Lattice Systems", LANL e-print http://xxx.lanl.gov/abs/math.DS/0207121

[4] O. Bratteli, D. Robinson, Operator Algebras and Quantum Statistical Mechanics I, Springer, New York, 1979.

[5] F. Hiai, D. Petz, "The Proper Formula for Relative Entropy and its Asymptotics in Quantum Probability", Commun. Math. Phys, 143, 99-114, 1991.

[6] C. King, A. Leśniewski, "Quantum Sources and a Quantum Coding Theorem", J. Math. Phys, 39 (1), 88-101, 1998.

[7] K. Kraus, States, Effects, and Operations, Berlin, Germany: Springer-Verlag, 1983.

[8] M. Ohya, D. Petz, Quantum Entropy and its Use, Springer, Berlin, 1993.

[9] D. Petz, M. Mosonyi, "Stationary Quantum Source Coding", J. Math. Phys, 42, 4857-4864, 2001, http://xxx.lanl.gov/abs/quant-ph/9912103

[10] D. Ruelle, Statistical Mechanics, W.A. Benjamin, New York, 1969. 